\title{
Sensory ganglionopathy mediated by autoimmunity and toxicity
}

\author{
Tara Kannan ${ }^{1}$ and Adel Ekladious ${ }^{2 *}$ \\ ${ }^{1}$ Medical Student, School of Medicine and Public Health, University of Newcastle, Newcastle, NSW 2308, Australia \\ ${ }^{2}$ Internal Medicine Specialist, Faculty of Health and Medical Sciences, University of Western Australia, Perth, WA 6009, Australia
}

\begin{abstract}
Sensory ganglionopathy is a rare, disabling disease with varied aetiology. We present a young lady with jaundice and ataxia on a background of multiple autoimmune conditions and chronic use of over-the-counter medication containing vitamins B6 and E. A diagnosis of autoimmune hepatitis-related ganglionopathy was made. Intravenous immunoglobulin was commenced and, vitamins B6 and E were discontinued. Steroids and Azathioprine treatment was also commenced. The patient recovered completely with normalisation of liver function tests and nerve conduction studies.
\end{abstract}

\section{Sensory ganglionopathy mediated by autoimmunity and toxicity}

A 33-year Chinese nurse was referred by her general practitioner with a two-week history of jaundice and an ataxic gait. Her past medical history included Graves' disease diagnosed three years ago and treated with radioactive iodine, Type 1 diabetes mellitus diagnosed at age 20 and treated with an insulin pump, and pernicious anaemia treated with regular cyanocobalamin injections.

On examination, she was deeply jaundiced with no signs of chronic liver disease or Graves' disease such as ophthalmopathy, dermopathy (pretibial myxoedema) or clubbing. Abdominal examination revealed palpable tender hepatomegaly but no splenomegaly or ascites. Examination of the shins revealed bilateral necrobiosis lipoidica.

Blood tests showed haemoglobin $14 \mathrm{~g} / \mathrm{dL}(12-15.5 \mathrm{~g} / \mathrm{dL})$, alanine aminotransferase $300 \mathrm{U} / \mathrm{L}$ (7-55 U/L), aspartate transaminase 200 U/L (8-48 U/L), alkaline phosphatase $265 \mathrm{U} / \mathrm{L}$ (40-129 U/L), gammaglutamyl transferase $200 \mathrm{U} / \mathrm{L}(8-61 \mathrm{U} / \mathrm{L})$, bilirubin $200 \mathrm{umol} / \mathrm{L}$ (3$17 \mathrm{umol} / \mathrm{L})$, direct bilirubin $150 \mathrm{umol} / \mathrm{L}(0-3 \mathrm{umol} / \mathrm{L})$ and indirect bilirubin $50 \mathrm{umol} / \mathrm{L}(3-14 \mathrm{umol} / \mathrm{L})$. She had normal albumin (3.4-5.4 $\mathrm{g} / \mathrm{dL})$, international normalised ratio $(<1.1)$ and platelets $(150,000$ $450,000 \mathrm{uL}$ ). Serology and polymerase chain reaction for hepatitis $\mathrm{A}, \mathrm{B}, \mathrm{C}, \mathrm{D}$ and $\mathrm{E}$ were negative. Globulin was elevated at $6 \mathrm{~g} / \mathrm{dL}(2.4$ to $4.1 \mathrm{~g} / \mathrm{dL}$ ). Antinuclear antibodies (ANA) were positive at 1:400, anti-mitochondrial antibodies were not detected, DNA binding and complements were normal and smooth muscle antibodies (SMA) was positive at 1:400. Iron studies, 24-hour urine for copper (10-30 ug), serum ceruloplasmin (14-40 mg/dL) and alpha one antitrypsin (100-300 $\mathrm{mg} / \mathrm{dL}$ ) were all either negative or normal. Antibodies to liver kidney microsome type 1 (anti-LKM1) and soluble liver antigen were positive. HBA1c $(<6 \%)$, serum B12 $(190-950 \mathrm{pg} / \mathrm{mL})$, methylmalonate $(0-0.40$ $\mathrm{umol} / \mathrm{L})$ and homocysteine levels $(<15 \mathrm{umol} / \mathrm{L})$ were normal. Liver ultrasound was unremarkable. Given the suspicion for autoimmune hepatitis, a liver biopsy was undertaken.

The patient then had two falls during the day and felt unsteady on her feet. A repeat examination excluded encephalopathy. An urgent magnetic resonance imaging (MRI) of brain completed with and without gadolinium ruled out stroke or space occupying lesion. Serum ammonia was $14 \mu / \mathrm{dL}$ (normal $15-45 \mu / \mathrm{dL}$ ). Repeat liver function tests were unchanged.

An urgent neurology referral was made. Examination revealed intact cranial nerves apart from an absent blink reflex bilaterally. Globally, tone and strength were normal but reflexes (biceps, triceps, brachioradialis, knee, and ankle) were diminished. There was impaired proprioception and vibration globally, athetoid movements in the arms and an impaired heel-shin test in the legs.

An urgent cerebrospinal fluid (CSF) study excluded Guillain-Barré syndrome, showing instead normal protein with no cells or oligoclonal bands. A nerve condition study (NCS) showed markedly reduced sensory nerve action potentials (SNAPs) of radial, median, ulnar, sural and superficial peroneal nerves in a non-length dependent pattern (Table 1). There was normal motor action potential and normal conduction. Needle electromyography (EMG) showed normal compound muscle action potential. MRI spine showed T2 weighted hyperintensity in the posterior cervical cord. Skin biopsy findings revealed degeneration of somatic unmyelinated small fibres in a non-length dependent fashion

Table 1. Sensory nerve conduction

\begin{tabular}{|c|c|c|c|c|c|c|c|c|c|}
\hline \multicolumn{2}{|c|}{$\operatorname{Median}(\geq 8 \mathrm{uV})^{\dagger}$} & \multicolumn{2}{|c|}{$\operatorname{Ulnar}(\geq 5 \mathrm{uV})$} & \multicolumn{2}{|c|}{ Radial $(\geq 15 u V)$} & \multicolumn{2}{|c|}{ Sural $(\geq 5 u V)$} & \multicolumn{2}{|c|}{$\begin{array}{c}\text { Superficial } \\
\text { Peroneal }(\geq 6 \mathrm{uV})\end{array}$} \\
\hline $\mathrm{Rt}$ & $\mathrm{Lt}$ & $\mathrm{Rt}$ & $\mathrm{Lt}$ & $\mathrm{Rt}$ & $\mathrm{Lt}$ & $\mathrm{Rt}$ & $\mathrm{Lt}$ & $\mathrm{Rt}$ & $\mathrm{Lt}$ \\
\hline$\downarrow \downarrow \downarrow$ & $\downarrow \downarrow \downarrow$ & $\downarrow \downarrow \downarrow$ & $\downarrow \downarrow \downarrow$ & $\downarrow \downarrow \downarrow$ & $\downarrow \downarrow \downarrow$ & $\downarrow$ & $\downarrow \downarrow$ & $\downarrow$ & $\downarrow \downarrow$ \\
\hline
\end{tabular}

Rt: right; Lt: left

'Normal values in parentheses.

${ }^{*}$ Correspondence to: Associate Professor Adel Ekladious, Faculty of Health and Medical Sciences, University of Western Australia, G11 M Block, UWA Health Campus (QE11), Monash Avenue, Nedlands 6009, Australia, Tel: 61499449905; E-mail: ekladiou@gmail.com

Keywords: sensory ganglionopathy, autoimmune hepatitis, ataxia, pyridoxine toxicity Received: June 21, 2021; Accepted: June 28, 2021; Published: June 30, 2021 
and decreased intraepidermal small nerve fibre densities. Repeat blood tests demonstrated normal methylmalonic acid, homocysteine, B12, folate, zinc and copper.

An initial diagnosis of peripheral neuropathy was made, and Duloxetine was commenced with no improvement. After further consultation with an electrophysiologist neurologist, the diagnosis was revised to acute sensorineural ganglionopathy. As advised by the neurologist, the patient was commenced on intravenous immunoglobulin. The liver biopsy showed an abundance of plasma cells in the port hepatis, in addition to rosette formation, centrilobular hepatocellular necrosis and mononuclear cells. This was consistent with autoimmune hepatitis. With the guidance of the neurology and hepatology teams, Prednisolone $1 \mathrm{mg} / \mathrm{kg}$ daily was started for six weeks with close follow up including blood testing. The patient then disclosed chronic over-the-counter medication use in the form of vitamin $\mathrm{E}$ and B6 (pyridoxine) 2g/day. These were both discontinued. By week four, the patient's ataxia improved. Prednisolone was then reduced to $30 \mathrm{mg}$ daily with gradual reduction of the steroid dose by $2 \mathrm{mg}$ every week and Azathioprine was started at $1.5 \mathrm{mg} / \mathrm{kg}$ as a steroid sparing agent. By week ten, the liver function tests were normal and a repeat NCS showed resolution of ganglionopathy with almost normal SNAPs. The patient remained on Azathioprine for six months with gradual reduction in dosing until complete cessation.

\section{Discussion}

Sensory ganglionopathy or sensory neuronopathy is a rare yet disabling disorder caused by degeneration of the dorsal root ganglia (DGR) or with trigeminal sensory neurons [1]. Sensory ganglia comprise cell bodies of afferent neurons which transmit sensory input from the periphery to the central nervous system. The vascularisation of DRG is unique in that capillaries are fenestrated and there is a lack of tight junction proteins, thus forming a permeable blood-nerve barrier [1]. As a result, the DRG is vulnerable to blood-borne molecules, such as, proteins, antibodies, toxins and lymphocytes.

There are several features that help differentiate between a ganglionopathy and a peripheral neuropathy [1]. Ganglionopathies are confined to sensory neurons and hence, strength is spared. Ganglionopathies are also not dependent on the length of axons, allowing asymmetric sensory loss which can start in any territory such as the scalp, face and proximal limbs. This differs from the more common length-dependent pattern of peripheral neuropathies wherein symptoms develop symmetrically at the terminals of the longest nerves, often affecting the feet before ascending proximally. Symptom onset of ganglionopathy is more rapid than peripheral neuropathy, typically evolving over days or weeks. Clinically, ganglionopathies can affect either the large or, less commonly, small nerve fibres - the former being more common. Large fibre signs include impaired sense of touch, vibration, proprioception, Romberg's and tendon reflexes. Pseudo-athetosis reflects a severe loss of proprioception. When small fibres are affected, examination is unremarkable. Ataxia is an early feature of ganglionopathy since proximal projections of ganglia form posterior spinal roots containing spinocerebellar fibres. In addition, dysautonomia stemming from degeneration of autonomic ganglia may present as orthostatic hypotension, arrhythmia, gastric dysmotility and sweating.

Ganglionopathy has varied aetiologies. A study of 148 patients with ganglionopathy reported the following rates for underling aetiologies: idiopathic (33\%), paraneoplastic (28\%), autoimmune (20\%), toxic (11\%), inherited (5\%), and infectious (1\%) [2]. Small cell lung cancer is most often implicated in paraneoplastic ganglionopathy, followed by breast, ovarian, prostate, colon and gastric cancer. Ganglionopathy often precedes a cancer diagnosis by 3-8 months and anti-Hu antibodies may be positive [3]. Autoimmune ganglionopathies are caused by Sjogren's syndrome, systemic lupus erythematous, autoimmune hepatitis and celiac disease. Toxic ganglionopathies can be due to platinum-based chemotherapeutic drugs, such as cisplatin, carboplatin and oxaliplatin, or vitamin B6 (pyridoxine) intoxication. Infectious ganglionopathies include human immunodeficiency virus, human T-lymphotropic virus type 1, enterovirus, Zika virus, EpsteinBarr virus and varicella-zoster. Inherited ganglionopathies are linked to Friedreich's ataxia, mitochondrial disorders and other novel genetic disorders. After extensive evaluation, up to $50 \%$ of patients have idiopathic ganglionopathy [3].

Our patient had dual aetiologies for her ganglionopathy autoimmune hepatitis and vitamin B6 toxicity. Of note, this patient also had five autoimmune conditions those being Graves' disease, Type 1 diabetes mellitus, pernicious anaemia, autoimmune hepatitis and autoimmune ganglionopathy. Autoimmune hepatitis (AIH) is characterised by progressive hepatocellular inflammation and necrosis. ${ }^{4}$ AIH is commonly seen in young females (7-8:1) and Caucasians [4]. Diagnosis requires biochemical findings of liver inflammation, seropositivity for SMA, ANA, and anti-LKM1, along with, histology findings of interface hepatitis. $1 \%$ of AIH patients may develop ganglionopathy [4]. The underlying mechanism is thought be a shared epitope between AIH and ganglionopathy. FGFR3, a protein for neuronal migration signalling, may be involved. ${ }^{4}$ In the case of liver involvement, FGFR3 expression is upregulated. Indeed, high titres of anti-FGFR3 antibodies were found in AIH-related ganglionopathy patients in one study [4]. To date, nine cases of AIH-related ganglionopathy have been reported in literature [4-8]. Our patient also used over the counter vitamin B6 at a toxic dose of 2g/day. Vitamin B6 (pyridoxine) intoxication is another form of toxic ganglionopathy. It is an essential vitamin that can also be used to prevent isoniazid toxicity and treat homocystinuria and pyridoxine-dependent epilepsy. Doses above $50 \mathrm{mg} /$ day is a risk factor and pyridoxine levels can be measured to assess toxicity [9]. In our patient, immunosuppressive treatment, along with, discontinuation of vitamin B6 was important to treat her ganglionopathy.

Investigations for all ganglionopathies comprise autoimmune studies, computer tomography and potentially biopsy for malignancy [1]. CSF analysis can reveal elevated protein, pleocytosis or oligoclonal bands. NCS may initially be normal [1]. Later, there is reduced amplitudes of SNAPs. A hallmark of non-length dependent pattern is when SNAPs are reduced in the arms (radial, median, ulnar) or the blink reflex is absent (trigeminal sensory neuron); while, SNAPs in the lower legs are normal [1]. In contrast, peripheral neuropathies mostly affect the lower extremities first. Blink reflex testing can also help differentiate between paraneoplastic and non-paraneoplastic causes as it is absent in the latter [10]. In small fibre ganglionopathies, SNAPs are normal. Needle EMG may have abnormal activity. MRI is sensitive and findings include T2 hyperintensity in the posterior column [1]. Findings from MRI and NCS can be virtually diagnostic of ganglionopathy. Further testing can include sural nerve biopsy showing loss of large fibres and skin biopsy showing a non-length dependent pattern. Definitive testing is with DRG biopsy, but this is invasive and rarely done. Findings include neuronal loss, the Nageotte nodules and mononuclear infiltrates [11].

Camdessanché, et al. proposed diagnostic criteria for ganglionopthy [2]. These comprise clinical components, such as ataxia, asymmetrical 
distribution and non-length dependent pattern, along with, electrophysiological components, such as, absence of one or reduction of three SNAPs, and less than two nerves with abnormal motor NCS.

Treatment of ganglionopathy is with immunotherapies such as glucocorticoids, immunosuppressive agents, plasma exchange, intravenous immunoglobulin and rituximab. ${ }^{1}$ Paraneoplastic ganglionopathy often does not resolve after cancer treatment. Early initiation of treatment is critical given the narrow therapeutic window before irreversible damage to the DRG when patient can become wheelchair-bound or bedridden. In one study, improvement occurred if treated within two months of symptom onset; while, disease stabilisation occurred if treated within eight months [12].

Sensory ganglionopathy is a rare disorder with varied aetiologies characterised by an asymmetric, non-length dependent pattern of sensory loss. The presence of sensory ataxia, especially in the context of systemic disease, should prompt investigation for ganglionopathy. If ganglionopathy is confirmed, clinicians are advised to consider an underlying organ specific autoimmune disease. Nevertheless, diagnosing ganglionopathy due to autoimmunity should not preclude investigation to exclude other causes such as malignancy, toxicity and infection. Of significance, timely identification of the cause of the ganglionopathy is paramount, as early treatment can prevent complete loss of DRG neurons, and can even lead to complete cure, as was the case in our patient.

\section{Acknowledgements}

\section{Funding}

None.

\section{Conflict of interest}

None.

\section{References}

1. Amato AA, Ropper AH (2020) Sensory Ganglionopathy. N Engl J Med 383: 1657 1662. [Crossref]

2. Camdessanché JP, Jousserand G, Franques J, Pouget J, Delmont E, et al. (2012) A clinical pattern-based etiological diagnostic strategy for sensory neuronopathies: a French collaborative study. J Peripher Nerv Syst 17: 331-340. [Crossref]

3. Gwathmey KG (2016) Sensory neuronopathies. Muscle Nerve 2016; 53:8-19. [Crossref]

4. Martinez AR, de Lima FD, Martins MP, Pereira IE, Miotto N, et al. (2020) Sensory neuronopathy is a specific and disabling neurological manifestation of autoimmune hepatitis. Eur J Neurol 27: 2072-8. [Crossref]

5. Magy L, Bassez G, Chassande B, Poynard T, Léger JM (1997) Sensory neuronopathy associated with chronic autoimmune hepatitis. Rev Neurol 153: 70-72.

6. Merchut MP, Adams EM, Morrissey M (1993) Sensory neuronopathy in autoimmune chronic active hepatitis. Neurology 43: 2410-2411. [Crossref]

7. Liedholm LJ, Månsson A, Holmgren H (1994) Subacute sensory neuropathies. Nord Med 109: 296-297. [Crossref]

8. Lauria G, Pareyson D, Grisoli M, Sghirlanzoni A (2000) Clinical and magnetic resonance imaging findings in chronic sensory ganglionopathies. Ann Neurol 47:104-109. [Crossref]

9. Echaniz-Laguna A, Mourot-Cottet R, Noel E, Chanson JB (2018) Regressive pyridoxine-induced sensory neuronopathy in a patient with homocystinuria. BMJ Case Rep 2018: bcr2018225059. [Crossref]

10. Auger RG, Windebank AJ, Lucchinetti CF, Chalk CH (1999) Role of the blink reflex in the evaluation of sensory neuronopathy. Neurology 53: 407-408. [Crossref]

11. Fargeot G, Echaniz-Laguna A (2021) Sensory neuronopathies: new genes, new antibodies and new concepts. J Neurol Neurosurg Psychiatry 92: 398-406. [Crossref]

12. Antoine JC, Robert-Varvat F, Maisonobe T, Créange A, Franques J, et al. (2016) Identifying a therapeutic window in acute and subacute inflammatory sensory neuronopathies. J Neurol Sci 361:187-191. [Crossref]

Copyright: @2021 Kannan T. This is an open-access article distributed under the terms of the Creative Commons Attribution License, which permits unrestricted use, distribution, and reproduction in any medium, provided the original author and source are credited. 